\title{
Timing of Oversedation Events Following Opiate Administration in Hospitalized Patients
}

\author{
John S. Garrett ${ }^{\mathrm{a}, \mathrm{d}}$, Annelise Vanston ${ }^{\mathrm{a}}$, Hoa L. Nguyen ${ }^{\mathrm{b}}$, \\ Cindy Cassity ${ }^{\mathrm{a}}$, Angela Straza ${ }^{\mathrm{c}}$
}

\begin{abstract}
Background: Unintended overdoses of opiate medications are potentially lethal events. Monitoring patients for oversedation is fundamental to ensuring safe use of opiates, and the timing of this evaluation is guided by the onset of action, time to max effect and duration of action of the opiate. The study's aim was to describe the timing of oversedation in relation to the predicted duration of action of the administered opiate.
\end{abstract}

Methods: This study was conducted as a retrospective review of all opiate-related oversedation events during a 2-year period involving patients admitted to an urban teaching hospital.

Results: Of the 53 opiate-related oversedation events evaluated, 47\% occurred after the predicted maximal duration of action of the administered opiate.

Conclusion: Opiate-induced oversedation routinely occurs after predicted based upon duration of action. The study findings have profound implications upon nursing practice regarding duration of time required to monitor for opiate-induced oversedation.

Keywords: Oversedation; Opiate; Patient safety

\section{Introduction}

Effective pain management for patients suffering while in the hospital is a priority for regulatory agencies, healthcare pro-

Manuscript submitted April 1, 2021, accepted April 15, 2021

Published online May 25, 2021

aDepartment of Healthcare Quality and Patient Safety, Baylor University Medical Center, Dallas, TX, USA

bDepartment of Population and Quantitative Health Sciences, University of Massachusetts Medical School, Worcester, MA, USA

'Department of Pharmacy, Baylor University Medical Center, Dallas, TX, USA

${ }^{\mathrm{d} C o r r e s p o n d i n g ~ A u t h o r: ~ J o h n ~ S . ~ G a r r e t t, ~ D e p a r t m e n t ~ o f ~ H e a l t h c a r e ~ Q u a l-~}$ ity and Patient Safety, Baylor University Medical Center, Dallas, TX, USA. Email: John.garrett@bswhealth.org

doi: https://doi.org/10.14740/jocmr4498 viders and patients [1]. Opioid analgesia remains a primary pharmacologic intervention for managing pain in hospitalized patients [2]. Sedation, or decreased level of consciousness beyond baseline, is a common secondary effect of opiates affecting over $60 \%$ of patients. As sedation progresses, lifethreatening respiratory depression and failure occur, affecting $0.04-2 \%$ of patients overall $[3,4]$. Although relatively uncommon, opioid-induced respiratory depression is a leading cause of preventable patient death in the United States primarily due to the vast number of patients who receive opiate pain medications [5-8]. A core tenet to the safe use of opiates is monitoring patients for the development of sedation and respiratory failure [2]. This evaluation is often accomplished through the use of standardized sedation assessment scores and hemodynamic monitoring to evaluate respiratory effort, blood oxygen content, and/or end tidal $\mathrm{CO}_{2}$. Monitoring practices are inconsistently applied and the equipment needed for continuous monitoring is not available on all floors of hospitals.

The American Society for Pain Management guidelines for monitoring of opioid-induced sedation and respiratory depression advise that patient be evaluated for sedation after the administration of opiates [9]. In clinical practice, the timing of this evaluation is guided by the onset of action, time to max effect and the predicted duration of action of these medications. Individual patient conditions such as impaired hepatic and renal clearance, body habitus and dose stacking may impact the duration of action of the medications. This can make it difficult, if near impossible, for clinicians to identify patients at risk for oversedation and respiratory depression [10]. In the setting of lack of continuous monitoring, sedation and respiratory assessments must be guided by the timing of opiate administration, time to peak effect and duration of action.

The aim of this work was to describe the timing of oversedation event in relationship to the administration of opiate medications, the onset of activity and duration of action.

\section{Materials and Methods}

\section{Study population and setting}

This was a retrospective study reviewing a pre-existing registry of oversedation events which occurred at Baylor University Medical Center, a large urban teaching hospital, between the dates of July 1, 2016 and June 30, 2018. 


\section{Patients' eligibility and the study outcome}

An oversedation event was defined as an occurrence of sedation or respiratory depression severe enough that the primary care team felt emergent reversal with naloxone was indicated. Cases in which naloxone was administered were individually reviewed by study staff to ensure naloxone administration successfully reversed the sedation event or that opiate medication was felt to be the causative etiology of sedation. An oversedation case was confirmed by one of the following: 1) successful reversal of sedation or 2) there is evidence the patient suffered a hypoxic encephalopathy due to the oversedation event or 3) the patient was pronounced dead due to presumed sedation were included in the registry.

Patients were excluded from the registry if reversal with naloxone was 1) not successful or 2) the patient did not have evidence of hypoxic encephalopathy due to a suspected oversedation event or 3) received naloxone during the course of procedural sedation. Patients who received an opiate through patient-controlled analgesia (PCA) were excluded from the study as they received small doses of opiates and the calculation of onset of activity and duration of action was not available. Additionally, patients were excluded if they were under 18 years of age, prisoners, pregnant, with hospice disposition, with donot-resuscitate orders.

\section{Data collection}

Information on patients' characteristics (age and body mass index (BMI)) and medical history (e.g., obstructive sleep apnea, pre-existing pulmonary disease, organ failure, smoking history and surgery duration) was collected. The timing of the oversedation event was determined by calculating the difference between the opiate administration time and the administration time of naloxone. The timing of the oversedation event was then compared to the predicted duration of action of the opiate. The predicted onset of activity and maximal duration of action was dependent upon both route of administration and type of drug. This reference information was pulled from the Department of Health and Human Services resource page as a general standard reference available to healthcare providers [11].

\section{Data analysis}

The study patients were categorized into two groups (yes/no) based upon if the oversedation event occurred within the expected duration of activity. Patients' demographic characteristics and medical history were presented as means and standard deviation (SD) and compared between the two groups using $t$-tests for continuous variables and percentages and compared using Chi-squared or Fisher exact tests for categorical variables.

The timing of opiate-induced oversedation was described using histogram. To estimate the timing of opiate-induced oversedation for each patient group, unadjusted and multivariable variable linear regression models adjusted for patient's age, sex, BMI and comorbidities were performed. All the anal- yses were performed using STATA 14.0 (StataCorp., College Station, TX).

This study was approved by the IRB and received a waiver of informed consent using a formal protocol and appropriate information security precautions in accordance with institutional review board regulations

\section{Results}

\section{Study patients' characteristics}

Overall, 269 inpatients received naloxone during the study period. Upon further review, 182 of these patients had no response to naloxone, opiates were not felt to be the cause of oversedation, or they were suspected of taking an outside opiate while in the hospital (nine) leaving 78 patients who received naloxone due to confirmed opiate-induced oversedation. Once the 15 patients who received their opiate via a PCA pump were excluded, 53 patients were available for the final analysis. Of the 53 patients who met the study eligibility criteria, $46 \%$ (25) were found to be oversedated and received naloxone after the opiate was predicted to have worn off. Patients' demographics and medical history for the two groups are presented in Table 1.

The proportion of patients with renal insufficiency $(32 \%)$ or liver disease $(16 \%)$ in the cohort who remained oversedated after the opiate should have worn off, which would impact clearance of the opiate and could explain the prolonged duration of action, was higher than the general population but was no different than the group who were oversedated within the predicted duration of action of their respective opiate. Opiate naive patients, those who had not taken an opiate chronically prior to admission, were more likely to develop oversedation after the maximal duration of action rather than within the predicted duration of action ( $48 \%$ vs. $21 \%, \mathrm{P}<0.04$, Table 1$)$. There was not a significant difference between the type of opiate administered and the likelihood to develop oversedation after the maximum duration of action of the agent $(\mathrm{P}=0.42)$.

\section{Study outcome}

The timing of the oversedation event was then compared to the predicted duration of action. The difference between the predicted duration of action and timing of oversedation for the study population is described in Figure 1. The timing was significant longer in the "yes" group vs. the "no" group (Table 2). On average, the difference between the timing of oversedation and the predicted maximal duration of activity was $24 \mathrm{~min}$, though this ranged to as long as $10 \mathrm{~h}$ in three patients (Table 2).

\section{Discussion}

Prompt recognition of opiate-induced oversedation allows healthcare providers to rescue patients prior to the development of potentially fatal opiate-induced respiratory failure. Although technologic adjuncts such as continuous pulse oxi- 
Table 1. Patient Characteristics by Oversedation Event

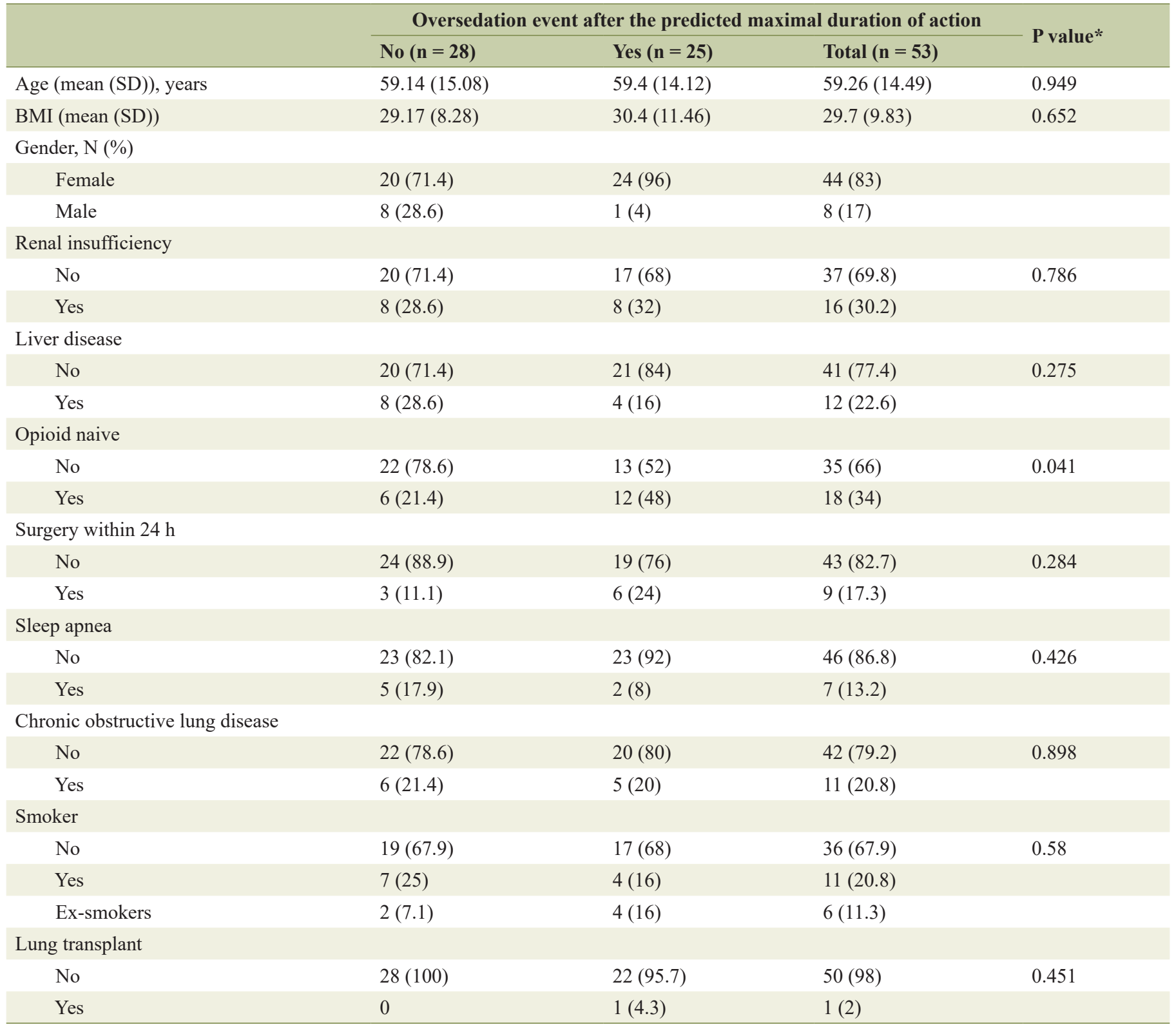

${ }^{*} \mathrm{P}$ values from $t$-tests for continuous variables and from Chi-squared or Fisher exact tests for categorical variables. BMI: body mass index.

metry and end tidal carbon dioxide assist the healthcare team in identifying opiate-induced respiratory depression, there are several drawbacks to relying upon this technology alone. The principal of these drawbacks is that these technologies are not available for every patient within a hospital. Patient care areas with continuous monitoring may still run into difficulties successfully using the technology. Alarm fatigue (where the healthcare team ignores alarms which fire frequently), poor patient compliance, outdated or limited equipment, and poor patient readings are ubiquitous and suggest other modalities are required to keep our patients safe.

Traditionally safe and effective monitoring has been addressed through standardized nursing assessments following the administration of a potentially sedating medication. Ideally this should occur after sufficient time has elapsed to allow the medication to reach maximal effect. In our study population, this post-opiate administration assessment was accomplished in $85 \%$ of patients who ultimately became oversedated. Monitoring may then occur on a regular basis until the medication has worn off and the sedative effects are not likely to be present. The exact time of onset of activity and maximal duration of action for each opiate and route of administration vary by source, but are widely published. When evaluating oversedation events within our hospital, we noted many (46\%) occurred well beyond the suspected timeframe in which the opiate should still be active. 


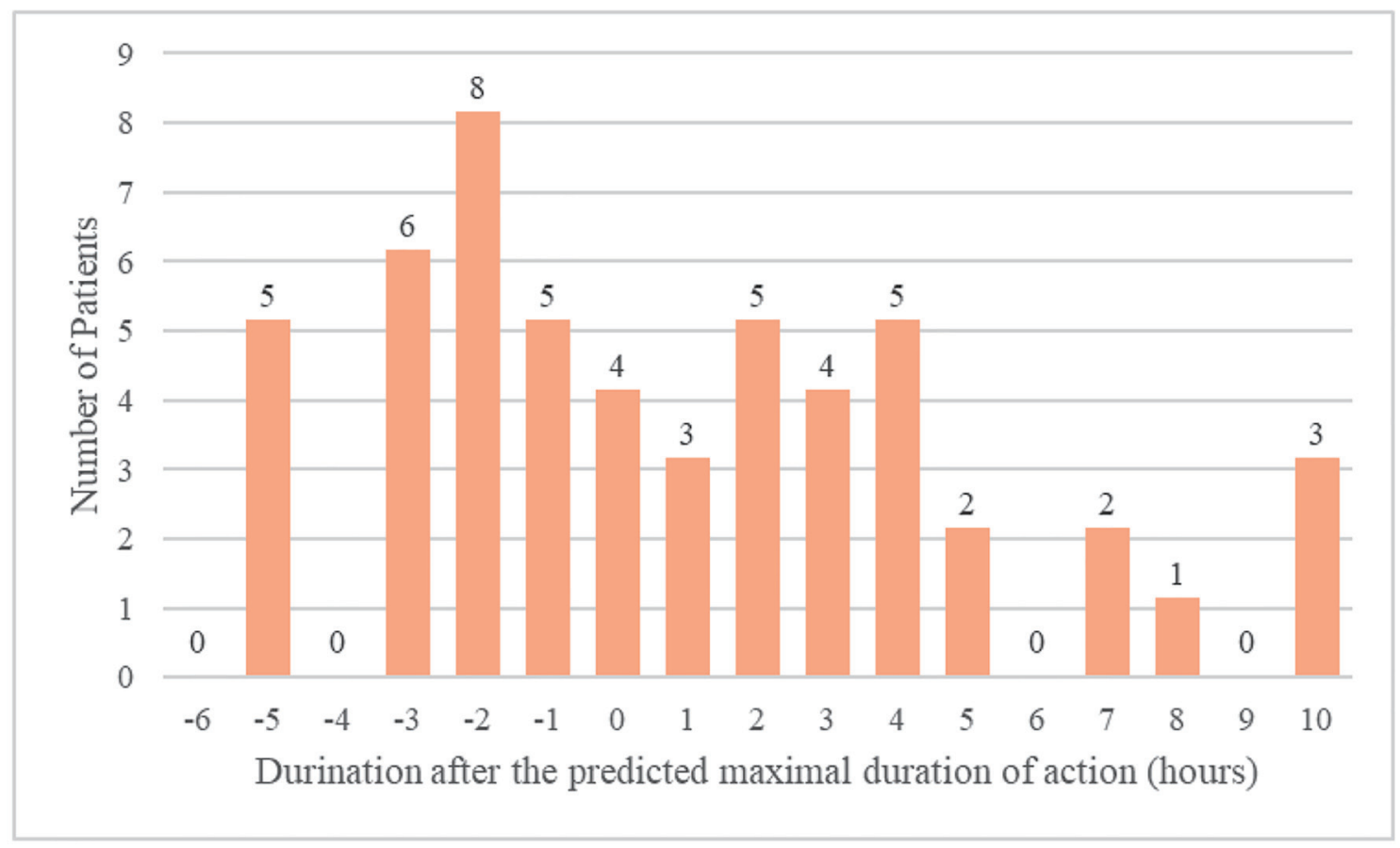

Figure 1. Difference between predicted duration of action and timing of oversedation.

In this study, we evaluated range in which patients had confirmed opiate-induced oversedation after the administration of an opiate. Surprisingly, we found several cases in which the patient was oversedated up to $10 \mathrm{~h}$ after the administration of an opiate. Overall, $46 \%$ of patients were found to be oversedated after the predicted duration of activity of the opiate had passed. This suggests that our primary modality for evaluating patients for oversedation may, in fact, miss almost half of the patients who will ultimately suffer from opiate-induced oversedation.

Patient conditions which may impact the clearance of opiates or other potentially sedating medications, such as renal insufficiency, cirrhosis, or obesity, did not appear more frequently in the group of patients who were found to be oversedated after the maximal duration of activity. Another etiology of prolonged effect included dose stacking and self-administration of opiates while in the hospital. In review of those cases with extremely prolonged duration of activity (greater than $6 \mathrm{~h}$ after the maximal duration should have passed), it does not appear the treating team felt self-administration of opiates was a likely etiology. We also evaluated the possibility that these patients became oversedated at night and were not identified until after morning rounds. Both incidence of oversedation and the dif- ference between oversedation timing and predicted maximal onset of duration of the opiate were evenly spread between morning, afternoon, and night. A more likely etiology to the prolonged effect of opiates includes dose stacking, which may occur in an occult manner on patients admitted to the hospital. Regardless of the etiology, it appears a significant portion of patients develop oversedation long after the expected duration of activity of the opiate they were administered.

The primary strength of this study is the number of oversedation events included in the database. Naloxone is administered liberally to inpatients with newly developed altered mental status, sedation, or respiratory depression. When reviewing these cases in detail, it appears the majority of naloxone administration does not reverse the sedation and is thus not the likely etiology of the oversedation. Though it is possible the dose of naloxone was not sufficient to fully reverse the opiate, this is felt unlikely.

There are several potential limitations inherent in this retrospective study design. It is possible the patients were administered opiates that were not charted in the healthcare record. The likelihood of this is low as our facility has tight diversion control standards and opiates are closely monitored. Second, it

Table 2. Estimates of OS After Maximal Duration of Action*

\begin{tabular}{|c|c|c|c|}
\hline & \multicolumn{2}{|c|}{$\begin{array}{l}\text { Mean OS after maximal dura- } \\
\quad \text { tion of action }(95 \% \mathrm{CI})\end{array}$} & \multirow{2}{*}{$\begin{array}{l}\text { Mean difference } \\
(95 \% \text { CI); P-value }\end{array}$} \\
\hline & No & Yes & \\
\hline Adjusted time after maximal duration of action, $\mathrm{h}$ & $-4.03(-8.41 ; 0.36)$ & $2.25(-2.16 ; 6.66)$ & $6.28(4.73 ; 7.83) ; \mathrm{P}<0.001$ \\
\hline
\end{tabular}

*Linear regression model adjusted for patient's age, sex, BMI and comorbidities listed in Table 1. OS: overall survival; Cl: confidence interval. 
is possible patients became oversedated but were not administered naloxone by the treating team. If this were to occur, the patient would not have fallen into study for evaluation. Third, we abstracted the oversedation time based upon the administration time of naloxone. During times of critical illness, staff often focuses on caring for the patient and then charts later. Though it is easy to correct the time of administration of naloxone in the healthcare record, it is possible that the nurse did not back time correctly. In order to evaluate this possibility, we evaluated the medical records of cases in which the oversedation occurred beyond the predicted maximal duration of action of the opiate. In each case, we found additional documentation (physician, registered nurse and rapid response team notes) which correlates with time the naloxone was administered.

\section{Conclusion}

Opiate-induced oversedation events frequently occur after the predicted maximal duration of activity of that opiate. The clinical team should be aware of the potential for sedation hours after opiate administration and should continue to monitor for oversedation.

\section{Acknowledgments}

The authors would like to acknowledge the support of the Baylor Healthcare System Foundation and to the efforts of Scott Ward, who continues to work upon the prevention of oversedation events in the hospital and facilitated this study.

\section{Financial Disclosure}

The study was funded through the Baylor Healthcare System Foundation.

\section{Conflict of Interest}

The authors have no conflict of interest to disclose.

\section{Informed Consent}

Not applicable.

\section{Author Contributions}

JSG contributed to this work through the conception and design, data collection, data analysis and interpretation, drafting the article, critical revision of the article, and final approval of the version to be published. AV contributed to this work through the conception and design, data collection, drafting the article, and final approval of the version to be published. HN contributed to this work through the data analysis and inter- pretation, drafting the article, and final approval of the version to be published. CC contributed to this work through critical revision of the article, and final approval of the version to be published. AS contributed to this work through the conception and design, critical revision of the article, and final approval of the version to be published.

\section{Data Availability}

The authors declare that data supporting the findings of this study are available within the article.

\section{References}

1. Joint Commission. Comprehensive accreditation manual for hospitals. Oak Brook, IL: Joint Commission. 2010.

2. Jarzyna D, Jungquist CR, Pasero C, Willens JS, Nisbet A, Oakes L, Dempsey SJ, et al. American Society for Pain Management Nursing guidelines on monitoring for opioid-induced sedation and respiratory depression. Pain Manag Nurs. 2011;12(3):118-145 e110.

3. Abou Hammoud H, Simon N, Urien S, Riou B, Lechat $\mathrm{P}$, Aubrun F. Intravenous morphine titration in immediate postoperative pain management: population kineticpharmacodynamic and logistic regression analysis. Pain. 2009;144(1-2):139-146.

4. Taylor S, Voytovich AE, Kozol RA. Has the pendulum swung too far in postoperative pain control? Am J Surg. 2003;186(5):472-475.

5. Cepeda MS, Farrar JT, Baumgarten M, Boston R, Carr DB, Strom BL. Side effects of opioids during short-term administration: effect of age, gender, and race. Clin Pharmacol Ther. 2003;74(2):102-112.

6. Weinger MB. Dangers of postoperative opioids. APSF Newsletter. 2006-22007;21:61-67.

7. Davies EC, Green CF, Taylor S, Williamson PR, Mottram DR, Pirmohamed M. Adverse drug reactions in hospital in-patients: a prospective analysis of 3695 patient-episodes. PLoS One. 2009;4(2):e4439.

8. The Joint Commission. Pain Assessment and management standards. R3 Report: Requirement, Rationale, Reference. 2017; (11)

9. Jungquist CR, Quinlan-Colwell A, Vallerand A, Carlisle HL, Cooney M, Dempsey SJ, Dunwoody D, et al. American Society for pain management nursing guidelines on monitoring for opioid-induced advancing sedation and respiratory depression: revisions. Pain Manag Nurs. 2020;21(1):7-25.

10. Brant JM, Stringer L, Jurkovich LR, Coombs NC, Mullette EJ, Buffington C, Herbert S, et al. Predictors of oversedation in hospitalized patients. Am J Health Syst Pharm. 2018;75(18):1378-1385.

11. The Texas Department of Health and Human Services. Onset, peak, and duration of common pain medications. Retrieved October 01, 2019. https://hhs.texas.gov/sites/ default/files/documents/doing-business-with-hhs/provider-portal/QMP/PainMedicationTable.pdf. 